\title{
Validity and Reliability of the Near-Death Experience Scale-6 (NDE-6)
}

\author{
Kevin P. Prosnick, Ph.D. \\ Center for Natural Medicine, Akron, Ohio \\ William J. Evans, Ph.D. \\ Westminster College, New Wilmington, PA
}

\begin{abstract}
This research reports the development and psychometric properties of a 6-item Near-Death Experience Scale (NDE-6) drawn largely from the 16-item parent scale. This short form evidenced adequate test-retest reliability and internal consistency, as well as construct validity. The index shows promise for use in research and as a screening scale in clinical contexts.
\end{abstract}

KEY WORDS: near-death experience; NDE Scale; NDE-6 Scale; transpersonal.

A near-death experience (NDE) has been defined as "a profound subjective event with transcendental or mystical elements" (Greyson, 1994, p. 103). Bruce Greyson's (1983) 16-item Near-Death Experience Scale (NDE-16) has been the preferred instrument in screening an individual or population in order to identify individuals who may have had a near-death experience. The NDE-16 exhibited adequate reliability in Greyson's (1983) sample. However, some of the items that comprise the scale have questionable predictive validity in that these items may not be measuring aspects unique to a NDE (Greyson, 1990).

The need for shortened versions of assessment instruments has increased in recent years with the increased importance of mental

Kevin P. Prosnick, Ph.D., is a licensed psychologist and clinical counselor in private practice, and William J. Evans, Ph.D., is Associate Professor of Education at Westminster College in New Wilmington, PA. Reprint requests should be addressed to Dr. Prosnick at 977 Hampton Drive, Akron, OH 44313; e-mail: kpp99@juno.com. 
health screening by health care providers, and the need for efficient methods to assess experiences of secondary interest. Short screening scales are also useful in surveys in order to select a subset of respondents who are likely to have had a particular experience or condition.

The purpose of the present study was to explore the psychometric properties of a 6-item scale drawn from the NDE-16 (Greyson, 1983). A parsimonious measure, shown to have adequate reliability and validity, would provide an efficient index with which to identify individuals who may have had NDEs.

\section{Methods}

This research examined the following reliability characteristics of the NDE-6: (a) internal consistency, and (b) test-retest reliability. With respect to construct validity, this research examined (a) the factor structure, and (b) the relationship of the index to measures of similar and different constructs. The institutional review board of Youngstown State University, where the study was conducted, approved the study; and all participants gave informed consent at the start of the study.

\section{Participants}

The sample consisted of 85 participants (65 women, 20 men) who were enrolled in a degree program at Youngstown State University, Youngstown, Ohio. The mean age was 36.76 years $(S D=9.64$, range $=$ 22 to 55). The mean number of years of education was $17.55(S D=1.40$, range $=16$ to 22). The racial composition of the sample was 94.1 percent Caucasian, 3.5 percent African American, 1.2 percent Hispanic American, and 1.2 percent unknown. The religious composition was 44.7 percent Catholic, 31.8 percent Protestant, 4.7 percent agnostic, 1.2 percent Jewish, 1.2 percent atheist, 15.3 percent other, and 1.2 percent unknown. The marital status of the sample was 63.5 percent married, 22.4 percent never married, 11.8 percent divorced, 1.2 percent cohabitating, and 1.2 percent unknown.

\section{NDE-6 Item Selection}

The goal of this stage of the project was to select a short subset of items from the NDE-16 (Greyson, 1983) that would most efficiently measure and screen for a NDE. The NDE-16 is a multiple choice questionnaire 
created to measure the occurrence of a NDE as well as assess four component areas of a NDE: cognitive, affective, paranormal, and transcendental. The four subscales that measure the four component areas have four items each. The scale has been used in several research studies (for example, Greyson, 1985, 1994; Pacciolla, 1996).

As alluded to in our introduction, five items (items 1, 5, 6, 7, and 9 on the NDE-16) may not measure facets unique to a NDE. For example, the rate of positive responses ("b" or "c" responses) to these five items among NDErs ranged from 66 percent to 92 percent (as contrasted to 20 percent to 86 percent for the remaining 11 items), whereas positive responses to these items among nonexperiencers ranged from 27 percent to 45 percent (as contrasted to only 2 percent to 17 percent for the other 11 items). The fact that more than a quarter of the nonNDErs chose at least a mildly positive response to these five items suggested to Greyson (1990) that these "items might therefore be regarded not as features unique to the NDE but as less specific responses to a neardeath encounter" (p. 160). Thus, these five items may be nonspecific responses for individuals who have had a close brush with death but not necessarily features that are unique to a NDE. Therefore, we considered these items, and the subscales they appear on, worthy of further study, but not deserving of inclusion on a short-form at this time.

Those five items are found on the cognitive, affective, and paranormal subscales of the NDE-16. Only the 4-item transcendental subscale does not contain any of the items in question. The NDE- 6 was created by combining the transcendental subscale with an item that measures the frequently reported out-of-body component of the NDE, plus a screening item. If this initial screening item is responded to in the negative, the participant is directed to stop responding.

A list of the items that comprise the NDE-6 is provided in the Appendix. For item 1, agreement with answer "c" receives 2 points, answer " $b$ " receives 1 point, and answer " $a$ " no points. For the remaining five items, agreement with answer "a" receives 2 points, answer " $b$ " receives 1 point, and answer " $c$ " no points. The instrument has a possible scoring range of 0 to 12 .

\section{Validity Instruments}

The Short Index of Self-Directedness (SISD). The SISD (Prosnick, Evans, and Farris, 2003) is an efficient 10-item Likert-type measure of the character trait of self-directedness. Individuals scoring high on 
the SISD can be characterized as more responsible, purposeful, resourceful, and self-disciplined. The SISD consists of items drawn from the 25-item Self-Directedness Scale of the Temperament and Character Inventory (TCI-125) (Cloninger, Przybeck, Svrakic, and Weltzel, 1994). Kevin Prosnick, Bill Evans, and Jaelyn Farris (2003) reported high one-week test-retest reliability $(r=.86)$ and high internal consistency $(r=.87)$ for the index. A significant positive correlation was found between the 25-item Self-Directedness Scale of the TCI-125 and scores on the SISD $(r=.77, \mathrm{p}<.001)$, thus indicating near equivalence. The NDE-6 was expected to evidence a positive and significant correlation, based upon previous research indicating that respondents who have had NDEs exhibit greater self-confidence and purposefulness (Greyson, 1994).

The Short Index of Present-Centeredness (SIPC). The SIPC is a 5item Likert-type scale intended to measure one's capacity to dwell in the present moment. Prosnick, Evans, and Elliott Ingersoll (Validity and reliability of the Short Index of Present-Centeredness [SIPC], unpublished manuscript, 2003) reported adequate one-week test-retest reliability $(r=.74)$ and average internal consistency $(r=.55)$. The SIPC evidenced construct validity by exhibiting significant positive correlations with measures of constructs that involve present-centered experience, that is, self-actualization and mystical experiences. Since present-centeredness is considered a subphenomena of self-actualization, and since previous research reported no significant relationship between an NDE and self-actualization (Greyson, 1992; Prosnick, 1999), the NDE-6 was expected not to evidence a significant relationship with the SIPC.

The Egotism/Transfluence Scale (ET Scale). The ET Scale (Prosnick, Validity and reliability of the Egotism/Transfluence Scale [ET Scale], unpublished manuscript, 2003) is a 30-item Likert-type scale created through multiple test construction methodologies, including factor analysis. The ET Scale is comprised of two subscales and one "honest reporting" validity-type item. The Egotism (Eg) Subscale, consisting of 11 items, measures the Gestalt resistance process whereby an individual mentally detaches from their experience and thereby avoids full interpersonal contact. The Transfluence (Tf) Subscale, consisting of 18 items, measures an individual's experience of transpersonal realities, including such experiences as seeing auras and mystical visions. Prosnick reported adequate internal consistency values of $r=.76$ and $r=.90$ for $\mathrm{Eg}$ and Tf respectively (1996), and 
high one-week test-retest reliability values of $r=.80$ for Eg and $r=.89$ for Tf (Validity and reliability of the Egotism/Transfluence Scale [ET Scale], unpublished manuscript, 2003). The Egotism Subscale exhibited construct validity by correlating positively with other Gestalt resistance processes and negatively with self-actualization, self-directedness, and overall life satisfaction; and the Transfluence Subscale evidenced construct validity by correlating positively with measures of report of mystical experiences, self-transcendence, and transpersonal self-concept (Validity and reliability of the Egotism / Transfluence Scale [ET Scale], unpublished manuscript, 2003). The NDE-6 was expected not to evidence a significant relationship with $\mathrm{Eg}$, since previous research reported no significant relationship between claim of an NDE and egotism (Prosnick, 1999). Also, since claim of an NDE was significantly correlated in a positive direction with transfluence in previous research findings (Prosnick, 1999), a significant positive correlation was expected between the NDE-6 and Tf.

Self-Expansiveness Level Form (SELF). The SELF (Friedman, 1983) is an 18-item Likert-type scale created through multiple test construction methodologies, including factor analysis. The Personal Subscale of the SELF (SELF-P), made up of five items, purports to measure the degree of self-identification that a person has with the "here-and-now" level of the self. The Transpersonal Subscale of the SELF (SELF-T), also made up of five items, purports to measure a person's "degree of identification with aspects of reality beyond that which is ordinarily conceived as being part of the individual" (Friedman, 1983, p. 40). Harris Friedman (1983) reported high two-month test-retest reliability values of $r=.83$ and $r=.80$ for the SELF-P and SELF-T respectively. Adequate internal consistency values of $r=.75$ and $r=.79$ for the SELF$\mathrm{P}$ and SELF-T respectively have been reported (MacDonald, Tsagarakis, and Holland, 1994). The SELF-P and SELF-T subscales have exhibited both construct and criterion validity (Friedman, 1983; MacDonald, Tsagarakis and Holland, 1994). Since "here-and-now" experience is considered a subphenomenon of self-actualization, and since previous research reported no significant relationship between an NDE and self-actualization (Greyson, 1992; Prosnick, 1999), the NDE-6 was expected not to evidence a significant relationship with the SELF-P. Since claim of an NDE was significantly correlated in a positive direction with measures of transpersonal experience in previous research findings (Prosnick, 1999), a significant positive correlation was expected between the NDE-6 and SELF-T. 
Physio-Kundalini Syndrome Index-7 (PKSI-7). "In Eastern spiritual traditions, the biological mechanism of both individual enlightenment and evolution of the species toward higher consciousness is called kundalini, a potential force that once awakened can produce a variety of mental, emotional, physical, and spiritual effects" (Greyson, 1993a, p. 278). The physio-kundalini syndrome is a specific nonpathological cluster of experiences that can be differentiated from psychiatric illness.

The PKSI-7 is a 7-item short-form drawn from the 19-item PhysioKundalini Syndrome Index (Greyson, 1993a). Prosnick and Evans (Validity and reliability of the Physio-Kundalini Syndrome Index-7 [PKSI-7], unpublished manuscript, 2003) reported good one-week testretest reliability $(r=.74)$ and average internal consistency $(r=.60)$. The PKSI-7 evidenced construct validity by exhibiting positive and significant correlations with measures of similar constructs: the Transfluence (Tf) Subscale, SELF-T, and the NDE-4. Greyson (1993b) reported that his sample of near-death experiencers "had shown definite signs of kundalini arousal" (p. 48). Therefore, a positive and significant correlation was expected between the NDE-6 and PKSI-7.

Near Death Experience-4 Scale (NDE-4 Scale). The NDE-4 is comprised of the four items from the transcendental subscale from the Near-Death Experience Scale-16 (Greyson, 1983). Greyson (1983) reported that the NDE-4 has adequate internal reliability $(r=.76)$ and excellent test-retest reliability $(r=.95)$. Regarding construct validity, the NDE-4 was highly correlated ( $r=.83$; Greyson, 1983) with the Weighted Core Experience Index (Ring, 1980), an instrument that purports to quantify the NDE experience. Greyson (1983) reported a mean of 3.85 and a standard deviation of $2.67(n=74)$ for the NDE-4 in a group of individuals claiming to have had a NDE. The NDE-6 was expected to exhibit a significant positive correlation with the NDE-4 because Greyson (1983) reported a positive and significant correlation between the NDE-4 and the NDE-16.

\section{Level of Significance}

In this research, $\alpha$ was set at .05 . Tests of construct validity in which a prediction was made (NDE-6 with SISD, Tf, SELF-T, PKSI-7, and NDE-4) employed one-tailed tests of significance, while tests in which significant relationships were not expected (NDE-6 with SIPC, Eg, and SELF-T) involved two-tailed tests. A correction for multiple 
simultaneous correlational tests was not applied, since each validity test was computed as a separate test of significance.

\section{Results}

As shown in Table 1, the NDE-6 had a mean of 3.08 and a standard deviation of 1.84 for the sample of 24 individuals who reported that they may have had an NDE (by answering " $b$ " or " $c$ " to question 1).

\section{Internal Consistency}

Question 1, the screening item that instructed participants who responded to it in the negative to stop responding, was omitted from computation of internal consistency so that a truer consistency of the NDE experience could be computed. For the remaining five items on the index, coefficient $\alpha$ was .71.

\section{Test-retest Reliability}

Temporal stability for a 7-day interval of a subsample of students $(\mathrm{n}=$ 16 ), who reported that they may have had a NDE, was $r=.89(\mathrm{p}<.000$, $\mathrm{df}=14)$. The mean for the first testing was $3.69(S . D .=2.50)$; for the second testing the mean was $3.50(S . D .=3.03)$. The means did not differ significantly, and therefore we concluded that there was no significant practice effect or regression to the mean in our sample.

\section{Construct Validity}

The scale was subjected to an exploratory principle components factor analysis utilizing an orthogonal rotation to the VARIMAX criterion, after excluding item 1 , the screening item. Those factors with eigenvalues greater than 1 were retained, which resulted in two factors. The structure coefficients of the five items on the two retained factors are given in Table 2. Interpretable, salient structure coefficients were considered to be .40 or greater. The first factor accounted for 39.8 percent of the variance, and is related to experience of and interaction with transpersonal entities in an unearthly or transcendent environment (items 4 and 5) during an NDE. The second factor, which accounted for 36.0 percent of the variance, is related to the onset (items 2 and 3 ) and culmination (item 6 ) of an NDE. 
Table 1

Statistics and Correlations Between NDE-6 and Other Study Variables (see text for variable acronyms)

\begin{tabular}{lrrrrr}
\hline \multicolumn{1}{c}{ Variable } & Possible Range & Mean & \multicolumn{1}{c}{$S D$} & $r_{N D E-6}$ & $p$ \\
\hline NDE-6 & $0-12$ & 3.08 & 1.84 & & \\
SISD & $10-50$ & 39.10 & 5.29 & .52 & $<.01$ \\
SIPC & $5-25$ & 16.92 & 3.03 & .24 & NS \\
Egotism & $11-55$ & 25.85 & 5.15 & -.27 & NS \\
Transfluence & $18-90$ & 45.63 & 11.97 & .30 & $<.05$ \\
SELF-P & $5-25$ & 22.18 & 3.41 & .21 & NS \\
SELF-T & $5-25$ & 14.55 & 4.69 & .29 & NS \\
PKSI-7 & $0-7$ & 1.25 & 1.34 & .41 & $<.05$ \\
NDE-4 & $0-8$ & 1.04 & 1.70 & .89 & $<.001$ \\
\hline
\end{tabular}

As shown in Table 1, the NDE-6 evidenced significant positive correlations with the SISD $(r=.52, \mathrm{p}<.01)$, Tf $(r=.30, \mathrm{p}<.05)$, the PKSI-7, $(r=.41, \mathrm{p}<.05)$ and the NDE-4 $(r=.89, \mathrm{p}<.001)$. No other significant correlations were found between scores on the NDE-6 and the other measures used in this study.

\section{Discussion}

The short index evidenced high temporal stability and high average internal reliability. There is a possible reason why the internal consistency coefficient reported was lower than the test-retest reliability coefficient. Cronbach's (1951) $\alpha$ coefficient of internal consistency is also a function of scale length (Anastasia, 1988), and the NDE-6 has only six items. Item heterogeneity can also weaken internal consistency. Examination of test-retest reliability can help to determine whether scale length and/or item heterogeneity is operating to weaken internal consistency, since test-retest reliability is not directly a function of scale length and/or item heterogeneity. The test-retest correlation for the NDE-6 for two test administrations one week apart was considerably higher than the internal consistency coefficient. Thus, scale length and/or item heterogeneity seemed to be lowering the internal consistency coefficient. Therefore, reliability of the NDE-6 can be considered adequate.

The current study employed an exploratory principle components factor analysis utilizing an orthogonal rotation to the VARIMAX 
Table 2

Factor Structure of the NDE-6

\begin{tabular}{|c|c|c|}
\hline \multirow[b]{2}{*}{ Item } & \multicolumn{2}{|c|}{ Factor } \\
\hline & 1 & 2 \\
\hline 1. Had a near-death experience? & \multicolumn{2}{|c|}{ (excluded) } \\
\hline 2. Felt separated from your physical body & -.14 & .83 \\
\hline 3. Entered some other, unearthly world & .56 & .67 \\
\hline 4. Encountered a mystical being or presence & $\underline{.88}$ & .02 \\
\hline 5. Saw deceased spirits or religious figures & .93 & .07 \\
\hline 6. Came to a border or point of no return & .16 & .82 \\
\hline
\end{tabular}

Note: Underscored values indicate salient and interpretable structure coefficients.

criterion. The results yielded two factors. Researchers attempting to cross validate these results might choose to use alternate factor analytic procedures. For example, principle axis factoring might be considered in addition to principle components analysis. Additionally, the orthogonal rotation utilized in this study assumed uncorrelated factors. However, because the underlying factors may be correlated, an oblique rotation may yield a different factor structure for the NDE-6.

As predicted, the NDE-6 evidenced significant positive correlations with the SISD, Tf, the PKSI-7, and the NDE-4. This is indicative of construct validity for the new index. The index evidenced a positive correlation with the SELF-T subscale, but the correlation coefficient was not significant. As also predicted, no other correlation differed significantly from zero. This indicates construct validity, and suggests that the scale may have discriminant validity.

A few limitations to the study should be mentioned. First, data came from one region of the United States and limited age range (22 to 55 years). Second, a small proportion of the respondents reported a possible NDE. Therefore, it would be prudent to replicate this study with larger and more varied samples. Third, the scale's items may be more sensitive to a positive or neutral NDE, and less sensitive to what is known in the literature as a frightening near-death experience or $f N D E$ (Bache, 1994, 1996).

Cut-off scores for the NDE-6 are considered provisional and tentative at this time, for two reasons: first, a small subset of individuals reported a possible NDE in our study $(n=24)$; and second, future research will 
need to assess the impact of our "screening item," which reflected more an opinion than an experience. Acknowledging these reservations, we suggest that a score of 0 or 1 point indicates a low probability of having had a NDE. Even so, in clinical contexts, individuals should be queried as to why they thought they might have had a NDE, to rule out an fNDE or an NDE with atypical features. A score of 2 or 3 (out of 12) suggests a moderate probability of having had a NDE. Such individuals could be administered the long version (the NDE-16), and/or a focused clinical interview could rule out an $\mathrm{NDEE}$ or atypical NDE, as above. A score of 4 or higher suggests a high probability of having had a NDE. Future research may determine if these cut-off scores offer sufficient levels of sensitivity, specificity, and predictive utility.

Finally, the short index is a more parsimonious and efficient method to screen for near-death experiences in epidemiologic and clinical studies than the NDE-16, due to its shorter length. The NDE-6 has an additional advantage over its predecessor. Whereas, the 16 -item version is usually given only when there is evidence that the individual or individuals may have had a NDE; the NDE-6, because of the inclusion of a screening item, can be given with a packet of other instruments to any population for either research or clinical purposes. If used clinically, the NDE-6 should be considered a screening scale only, and not a substitute for dialogue and exploration of that individual's possible NDE.

\section{Conclusion}

We have presented a screening scale for the self-report measurement of a near-death experience and documented the procedures utilized in the development of the scale. The scale is a short version of the 16-item Near-Death Experience Scale (Greyson, 1983). The screening scale is designed to measure lifetime history of having had a near-death experience. The scale may exhibit utility as a semi-continuous predictor for the probability of having had a NDE.

\section{References}

Anastasia, A. (1988). Psychological testing (6th ed.). New York, NY: Prentice Hall.

Bache, C. (1994). A perinatal interpretation of frightening near-death experiences: A dialogue with Kenneth Ring. Journal of Near Death Studies, 13, 25-45. 
Bache, C. (1996). Expanding Grof's concept of the perinatal: Deepening the inquiry into frightening near-death experiences. Journal of Near Death Studies, 15, 115-139.

Cloninger, C. R., Przybeck, T. R., Svrakic, D. M., and Weltzel, R. D. (1994). The Temperament and Character Inventory (TCI): A guide to its development and use. St. Louis, MO: Washington University/C. R. Cloninger.

Cronbach, L. (1951). Coefficient alpha and the internal structure of tests. Psychometrika, $16,297-334$.

Friedman, H. L. (1983). The Self-Expansiveness Level Form: A conceptualization and measurement of a transpersonal construct. Journal of Transpersonal Psychology, 15, $37-50$.

Greyson, B. (1983). The Near-Death Experience Scale: Construction, reliability, and validity. Journal of Nervous and Mental Disease, 171, 369-375.

Greyson, B. (1985). A typology of near-death experiences. American Journal of Psychiatry, 142, 967-969.

Greyson, B. (1990). Near-death encounters with and without near-death experiences: Comparative NDE scale profiles. Journal of Near Death Studies, 8, 151-161.

Greyson, B. (1992). Reduced death threat in near-death experiencers. Death Studies, 16 , $523-536$.

Greyson, B. (1993a). Near-death experiences and the physio-kundalini syndrome. Journal of Religion and Health, 32, 277-290.

Greyson, B. (1993b). The physio-kundalini syndrome and mental illness. Journal of Transpersonal Psychology, 25, 43-58.

Greyson, B. (1994). Near-death experiences and satisfaction with life. Journal of Near Death Studies, 13, 103-108.

MacDonald, D. A., Tsagarakis, C. I., and Holland, C. J. (1994). Validation of a measure of transpersonal self-concept and its relationship to Jungian and five factor model conceptions of personality. Journal of Transpersonal Psychology, 26, 175-201.

Pacciolla, A. (1996). The near-death experience: A study of its validity. Journal of Near Death Studies, 14, 179-185.

Prosnick, K. P. (1996). Final contact and beyond in Gestalt therapy theory and transpersonal research: A factor analytic study of egotism and transfluence (Doctoral dissertation, Kent State University, 1996). Dissertation Abstracts International, 5712, A5064 (UMI No. AAI9716995).

Prosnick, K. P. (1999). Claims of near-death experiences, Gestalt resistance processes, and measures of optimal functioning. Journal of Near-Death Studies, 18, 27-34.

Prosnick, K. P., Evans, W. E., and Farris, J. R. (2003). Development and psychometric properties of scores from the Short Index of Self-Directedness (SISD). Measurement and Evaluation in Counseling and Development, 36, 76-82.

Ring, K. (1980). Life at death: A scientific investigation of the near-death experience. New York, NY: Coward, McCann and Geoghegan.

\section{Appendix: Near-Death Experience Scale-6 (NDE-6)}

Please circle the answer to each question that best describes your experience.

1. Have you ever had a near-death experience?
a. No
b. Maybe; I'm not sure
c. Yes, I think I have 
If you answered "No" to question 1, please do not respond to items 2 to 6 below.

2. Did you feel separated from your physical body?
a. Clearly left the body and existed outside it
b. Lost awareness of the body
c. Neither

3. Did you seem to enter some other, unearthly world?
a. Clearly mystical or unearthly realm
b. Unfamiliar, strange place
c. Neither

4. Did you seem to encounter a mystical being or presence?
a. Definite being, or voice clearly of mystical or otherworldly origin
b. Unidentifiable voice
c. Neither

5. Did you see deceased spirits or religious figures?
a. Saw them
b. Sensed their presence
c. Neither

6. Did you come to a border or point of no return?
a. A barrier I was not permitted to cross; or "sent back" to life involuntarily
b. A conscious decision to "return" to life
c. Neither 